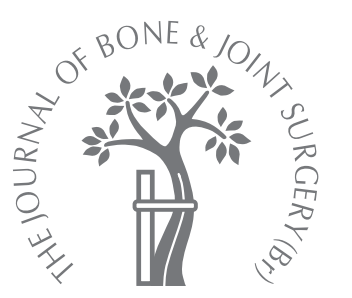

J. A. van der Sluijs,

L. de Gier,

J. I. Verbeke,

M.M.E.H. Witbreuk,

J. E. H. Pruys,

B. J. van Royen

From the VU

University Medical

Center, Amsterdam,

The Netherlands
I. A. van der Sluijs, MD, PhD, Orthopaedic Surgeon

M. M. E. H. Witbreuk, MD,

Orthopaedic Surgeon

- B. J. van Royen, MD, PhD,

Professor

- L. De Gier, MSc, Medical

Intern

Department of Orthopaedic

Surgery

J. I. Verbeke, MD, Paediatric

Radiologist

Department of Radiology

VU Medical Centre, Boelelaan

1117, 1007 MB Amsterdam, The

Netherlands.

J. E. H. Pruys, MD, PhD,

Orthopaedic Surgeon

Department of Paediatric

Orthopaedic Surgery

Wilhelmina Kinderziekenhuis,

Lundlaan 6, 3508 AB, Utrecht,

The Netherlands.

Correspondence should be sent to $\mathrm{Dr}$ J. A. van der Sluijs; e-mail: ja.vandersluijs@vumc.nl

(C)2009 British Editorial Society of Bone and Joint Surgery doi:10.1302/0301-620X.91B8 $21692 \$ 2.00$

$J$ Bone Joint Surg [Br] 2009;91-B:1090-3.

Received 27 August 2008;

Accepted after revision 23 April

2009

\title{
Prolonged treatment with the Pavlik harness in infants with developmental dysplasia of the hip
}

\begin{abstract}
We prospectively studied the benefits and risks of prolonged treatment with the Pavlik harness in infants with idiopathic developmental dysplasia of the hip. Bracing was continued as long as abduction improved. It was started at a mean age of four months (1 to 6.9). Outcome measures were the number of successful reductions, the time to reduction, the acetabular index and evidence of avascular necrosis at follow-up at one year.

In 50 infants with 62 subluxated and dislocated hips (42 Graf type III and 20 type IV), 37 were reduced successfully with a mean time to reduction of 13.4 weeks (SD 6.8). Bracing was successful in 31 type-III $(73.8 \%)$ and in only six type-IV hips $(20 \%, p=0.002)$. Avascular necrosis was seen in ten hips.

Prolonged treatment with the Pavlik harness for developmental dysplasia of the hip over the age of one month can be beneficial in type-III hips, but it is unclear as to whether this is the optimal treatment, since it may postpone the need for closed or open reduction to a more unfavourable age. The use of the Pavlik harness in type-IV hips in this age group is questionable, but if used, prolonged bracing is not advised.
\end{abstract}

Functional treatment in the Pavlik harness is a standard initial method of treatment for subluxated or dislocated hips in developmental dysplasia of the hip (DDH) in infants. ${ }^{1-3}$ Success has been reported in $80 \%$ to $95 \%$ of cases in several studies ${ }^{4-8}$ although others have given lower rates of between $50 \%$ and $80 \%{ }^{6,9-12}$ If treatment with the brace fails, closed or open reduction is indicated. ${ }^{1-3,13}$

The maximum period for use of the Pavlik harness is not known and until now, it has been unclear as to what extent prolonged bracing improves the rate of success. Some studies have suggested that long-term unsuccessful treatment with the brace is associated with a high rate of avascular necrosis (AVN) of the femoral head, deficiency of the posterior acetabulum, and/or deformity of the femoral head. ${ }^{5,7,14}$ In American practice, the recommended time in a Pavlik harness is a maximum of three to four weeks. ${ }^{1,2}$ In Europe this period is less defined. ${ }^{3}$

We postulated that, as long as abduction of the hip is improving, the continued use of the Pavlik harness may increase the number of successful reductions without the risk of AVN or residual dysplasia. In this prospective study we report the benefits and risks of prolonged bracing in Graf type-III and typeIV DDH. ${ }^{15}$

\section{Patients and Methods}

Between January 1999 and December 2006, infants with DDH were prospectively selected from all those referred to our institution by the local infant health centres for assessment of their hips, according to the national selective screening protocol. This recommends screening for DDH of infants between the age of three and five months who have one or more of the following: breech delivery, family history, leg-length discrepancy and limited abduction of the hip. The assessment was by clinical examination and standard ultrasonography using the Graf classification ${ }^{15}$ in which type-III is defined as a subluxated and type-IV as a dislocated joint.

Of 1641 infants referred for screening for idiopathic DDH, we diagnosed 52 who met the inclusion criteria (Graf III and IV). Two infants were excluded within the first two weeks of treatment because of a lack of compliance of the parents in one case and the wish for direct closed reduction by the parents in the other. Infants primarily treated elsewhere or those with syndromic dislocations were also excluded. We therefore studied 50 infants (all girls, 62 hips, 40 left, 22 right) who were treated with the Pavlik harness. There were 42 Graf type-III and 20 type-IV hips. The mean age at diagnosis and start of treatment was four months (1.1 to 6.9). 
Table I. Classification of avascular necrosis according to the Commission for the Study of Hip classification ${ }^{13}$

\begin{tabular}{lll}
\hline Grade & Radiological finding & Prognosis \\
\hline 1 & $\begin{array}{l}\text { Slightest degree of changes, femoral head ossification } \\
\text { centre with slightly blurred margins, slightly granular and } \\
\text { irregular in structure }\end{array}$ & Generally regresses over time \\
2 & $\begin{array}{l}\text { More blurred margins of the ossification centre with } \\
\text { irregular structure, more granular than for grade 1. Also } \\
\text { cyst formation or punched-out partial defects }\end{array}$ & $\begin{array}{l}\text { Changes usually regress, possible flattening of } \\
\text { the head }\end{array}$ \\
& $\begin{array}{l}\text { Whole femoral head ossification centre disintegrated or } \\
\text { only visible as individual fragments or a flat strip. Very small } \\
\text { head nucleus, possibly completely broken up or only visible } \\
\text { after many months }\end{array}$ & $\begin{array}{l}\text { Frequently, deformation of the femoral neck } \\
\text { inhich regress at a later date }\end{array}$ \\
& $\begin{array}{l}\text { Additional involvement of the epiphyseal plate. Irregularities } \\
\text { also apparent on the margins of the epiphyseal plate at the } \\
\text { femoral neck }\end{array}$ & $\begin{array}{l}\text { Serious consequences for growth } \\
4\end{array}$ \\
\hline
\end{tabular}

Although our screening protocol advises referral at three to five months, some children $(\mathrm{n}=9)$ were referred at a younger age because of severe limitation of abduction. No case was lost to follow-up.

The ultrasonographic examinations were performed or directly supervised by an experienced paediatric radiologist (JIV). Treatment was according to a standard protocol, in which the length of time in the harness was initially for 23 hours daily. ${ }^{1}$ The parents were instructed in the application and handling of the harness which was checked after one week in the outpatient clinic. Thereafter, all the infants were examined clinically at four to six weeks and by ultrasonography at intervals of six weeks. As long as abduction measured with the hips in $90^{\circ}$ of flexion increased, treatment with the harness was continued until a stable reduction was achieved. This was defined as symmetrical abduction and normal appearance on ultrasonography measured in slight flexion and no abduction. ${ }^{15}$ After reduction, use of the harness was continued at night for an additional six weeks.

In those infants in whom abduction initially increased to more than $45^{\circ}$, but then failed to improve during the following weeks, Pavlik bracing was supplemented by the use of a more rigid abduction device (Basko Healthcare, Hamburg, Germany). This was not applied when the abduction failed to improve with Pavlik bracing. We defined this treatment as Pavlik-Plus and both braces were worn together.

Treatment with the brace was discontinued when there was no increase in abduction over several weeks and no reduction on ultrasonographic examination. The failed infants then had traction for two weeks followed by closed or open reduction.

Standing radiographs of the pelvis were taken at followup at one year to assess any acetabular dysplasia and/or $\mathrm{AVN}$. The former was measured as the acetabular index (AI) and classified according to Tönnis ${ }^{16}$ as none, slight or severe, based on age-dependent normal values. The classification of AVN was according to the Commission for the
Table II. Relationship between the Graf type and the success of bracing

\begin{tabular}{lllllll}
\hline & & \multicolumn{2}{l}{ Pavlik } & & & \multicolumn{2}{l}{ Pavlik-Plus } \\
\cline { 3 - 4 } \cline { 6 - 7 } Graf type & Total & Success & Failure & & Success & Failure \\
\hline III & 42 & 24 & 8 & 7 & 3 \\
IV & 20 & $6^{*}$ & 8 & 0 & 6 \\
Total & 62 & 30 & 16 & 7 & 9 \\
\hline
\end{tabular}

* Fisher's exact test, $\mathrm{p}=0.002$

Study of Hip Dysplasia of the German Society for Orthopaedics and Traumatology ${ }^{13}$ which is a method used for the classification of AVN within the first years of life (Table I). Statistical analysis. The results were presented as the mean and SD. The significance of differences between interval type parameters was tested using the two sided $t$-test. For nominal data Fisher's exact test was used. The relation between the AI and the duration of treatment was described by the Pearson correlation coefficient. Statistical significance was defined as $\mathrm{p}<0.05$.

\section{Results}

Overall reduction was achieved in 37 of the 62 hips $(59.7 \%)$, 31 of 42 Graf type-III $(73.8 \%)$ but only six of 20 Graf typeIV $(30.0 \%$ Fisher's exact test, $\mathrm{p}=0.002$, Table II).

The mean total period of bracing, defined as the time to complete reduction, was 13.4 weeks (5 to 32). The time to reduction varied and success was time-dependent. Within the first six weeks, eight of 37 hips $(21.6 \%)$ were reduced, rising to 28 of $37(75.7 \%)$ hips after 18 weeks, with the last successful reduction occurring at 32 weeks. The time to reduction was not related to the Graf type. Time to reduction was 13.1 weeks for type-III (SD 7.1) and 14.7 weeks for type-IV hips (SD 5.5).

Pavlik-Plus treatment was prescribed in 16 infants, ten with type-III and six with type-IV. Successful reduction in these infants was achieved in seven of the ten with type-III at a maximum treatment period of 28 weeks. None of the 
Table III. Relationship between the Graf type, the effect of bracing and the outcome. The classification of avascular necrosis (AVN) (1 to 3 ) is according to commission for the study of hip classification and of dysplasia according to Tönnis ${ }^{13}$

\begin{tabular}{|c|c|c|c|c|c|c|c|c|c|}
\hline \multirow[b]{2}{*}{ Graf type } & \multirow[b]{2}{*}{ Number } & \multicolumn{4}{|l|}{ AVN } & \multirow[b]{2}{*}{ Mean (SD) acetabular index $\left({ }^{\circ}\right)$} & \multicolumn{3}{|c|}{ Number with dysplasia } \\
\hline & & Total & 1 & 2 & 3 & & Total & Slight & Severe \\
\hline III & 42 & & & & & & & & \\
\hline \multicolumn{10}{|l|}{ Total } \\
\hline Success Pavlik/Pavlik-Plus & & 28 & 3 & & & $24.7(3.9)$ & 21 & 6 & 4 \\
\hline Failed bracing. Closed (open reductions) & $8(3)$ & 9 & 1 & & 1 & $29(4.8)^{*}$ & 3 & 4 & 4 \\
\hline \multicolumn{10}{|l|}{ IV } \\
\hline Total & 20 & & & & & & & & \\
\hline Success with Pavlik/Pavlik-Plus & 6 & 5 & & 1 & & $24.5(5.1)$ & 3 & 3 & 0 \\
\hline Failed bracing. Closed (open reductions) & $6(8)$ & 10 & 1 & 1 & 2 & $29.7(7.4)$ & 6 & 2 & 6 \\
\hline Total & 62 & 52 & 5 & 2 & 3 & & 33 & 15 & 14 \\
\hline
\end{tabular}

type-IV hips was reduced successfully by the addition of a rigid abduction device (Table II). The decision to abandon bracing was made after five to 24 weeks in 25 hips $(40.3 \%)$. Closed $(n=14)$ or open reduction $(n=11)$ was performed at a mean age of 8.2 months (SD 1.9) and 9.5 months (SD 1.7), respectively.

At follow-up at one year, radiographs of the pelvis showed a normal AI in 33 of the 62 hips $(53.2 \%)$. Slight dysplasia was seen in $15(24.2 \%)$, and severe dysplasia in 14 hips (22.6\%, Table III). The duration of Pavlik bracing was not related to the AI correlation (Pearson correlation coefficient, $r=0.05)$. When comparing successful with failed Pavlik bracing, a normal AI was measured in 24 of 37 hips $(64.9 \%)$ after successful bracing, but in only nine of 25 hips $(36.0 \%)$ treated by closed or open reduction (Fisher's exact test, $\mathrm{p}=0.04$ ). The mean AI in the successful group $\left(24.7^{\circ}, 18^{\circ}\right.$ to $\left.32^{\circ}\right)$ was significantly lower than that in the failed group $\left(28.9^{\circ}, 16^{\circ}\right.$ to $39^{\circ}$, radiological $t$-test, $\mathrm{p}=0.005$ ).

Radiological signs of AVN were found in ten of the 62 hips (16.1\%). Five were grade 1, two grade 2 and three grade 3 (Table III). When combining all AVN grades, the length of Pavlik bracing was not related to the development of AVN since there was no difference in the mean length of bracing in both groups, $t$-test, $\mathrm{p}=0.54$. In addition AVN was not related to the Graf type $(\mathrm{p}=0.27)$ or to whether Pavlik bracing $(\mathrm{p}=0.29)$ was successful (Fisher's exact test). However, because of limited numbers, our study does not have the power to detect such differences.

\section{Discussion}

The most important finding of our study was that prolonged treatment with the Pavlik harness in DDH in infants can be effective and safe in some Graf type-III, but not in type-IV hips. With prolonged treatment we had a success rate of $73.8 \%$ in type III and $30 \%$ in type IV. An innovation of our study was the combined use of the clinical measurement of progressive hip abduction and ultrasonography in determining whether to continue treatment with the brace.
Successful bracing is time-dependent. A substantial number of reductions occur after four weeks, particularly in type III, in which we showed that only seven of 31 hips $(22.6 \%)$ were reduced within the first six weeks. By 12 weeks, half of the hips which eventually responded to bracing were not yet reduced. Consequently, a substantial proportion of hips would have been treated by closed or open treatment if we had followed the philosophy of limiting Pavlik bracing to four weeks.

Not only was the rate of successful reduction increased by prolonged Pavlik bracing but development of the hip, as defined by the prevention of residual dysplasia, also showed positive results. Development of the hip, as measured by the AI, proved not to be related to the duration of bracing, but to the Graf type and the success of Pavlik bracing.

A reasonable criticism is that our success rate using the Pavlik harness was only moderate and confirmed the reports of more cautious rates $(50 \%$ to $80 \%))^{6,9-12}$ Two factors may have been responsible. The age at the start of treatment may have influenced the final reduction rate. Our mean age of four months was twice that of studies reporting higher success rates. ${ }^{5,7}$ Ideally, bracing should start early; the later start in our study was a direct effect of the selective screening policy in The Netherlands which advises assessment of infants at risk at three to five months, although some with pronounced clinical features are referred earlier. Another factor may have been our high proportion of type-IV in relation to type-III hips. Others have shown that with severe dislocation, the success rates of Pavlik bracing declines and in type-IV hips, with a success rate of less than $50 \%$, Pavlik bracing is not undertaken. ${ }^{6,9,14}$ Therefore, the inclusion of type-IV hips in a series leads to a reduction of the overall rate of success of bracing.

We showed that the duration of bracing did not correlate with the development of AVN, which has a reported prevalence of $0 \%$ to $16 \%$, with the risk greatest in type-IV hips. ${ }^{14,17}$ Although there are no studies showing a relationship between the duration of brace treatment and the 
incidence of AVN, Suzuki et a ${ }^{17}$ found a rate of AVN of $12.3 \%$ after Pavlik bracing, with half of these infants being treated for no more than two weeks. The condition was also reported after closed or open reduction in hips which did not respond to Pavlik bracing ${ }^{3}$ and we also found that AVN occurred mainly in hips in which bracing had failed (Table III).

The Commission for the Study of Hip Dysplasia classification is sensitive and suitable for our age group ${ }^{13}$ in which the rate of AVN $(16 \%)$ was in agreement with that of Suzuki et al. ${ }^{17}$ Also, if grades I and II are excluded, the resultant rate of AVN of $5 \%$ corresponds to that of other reports. $^{6}$

A disadvantage of prolonged treatment with the brace in DDH is a delay in closed reduction in the case of failure. Also, closed reduction at increasing age may become more difficult, resulting in more open reductions, which may result in higher rates of AVN. In turn, delayed operations may lead to less time for remodelling.

We do not support the widespread practice of limiting Pavlik bracing to a maximum of three to four weeks in Graf type-III hips, in which we found that continuation in the harness was safe and effective as long as abduction improved. However, it is unclear as to whether prolonged bracing was the optimal policy since it may have delayed closed reduction and casting. The correct duration of Pavlik bracing in type-III cases remains to be determined. In type-IV hips in children older than one month, the use of the Pavlik harness is questionable but, if used, prolonged bracing is not advised.

\section{Supplementary material}

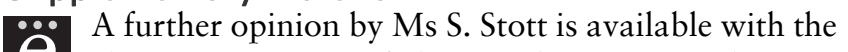
e electronic version of this article on our website at www.jbjs.org.uk
No benefits in any form have been received or will be received from a commercial party related directly or indirectly to the subject of this article.

\section{References}

1. Wenger DR. Developmental dysplasia of the hip. In: Wenger DR, Rang M, eds. The art and practice of children's orthopaedics. New York: Raven Press, 1993:256-96.

2. Staheli LT. Practice of pediatric orthopedics. First ed. Seattle: Lippincott Williams \& Wilkins, 2001:136-45.

3. Hefti F, Developmental dysplasia and congenital dislocation of the hip. In: Pediatric orthopaedics in practice. Berlin: Springer-Verlag, 2007:177-201.

4. Ramsey PL, Lasser S, MacEwen GD. Congenital dislocation of the hip: use of the Pavlik harness in the child during the first six months of life. J Bone Joint Surg [Am] 1976;58-A:1000-4.

5. Harris IE, Dickens R, Menelaus M. Use of the Pavlik harness for hip displacements: when to abandon treatment. Clin Orthop 1992;281:29-33.

6. Grill F, Bensahel H, Canadell J, et al. The Pavlik harness in the treatment of congenital dislocating hip: report on a multicenter study of the European Paediatric Orthopaedic Society. J Pediatr Orthop 1988;8:1-8.

7. Cashman JP, Round J, Taylor G, Clarke NM. The natural history of developmental dysplasia of the hip after early supervised treatment in the Pavlik harness: a prospective, longitudinal follow-up. J Bone Joint Surg [Br] 2002;84-B:418-25.

8. Nakamura J, Kamegaya M, Saisu T, et al. Treatment for developmental dysplasia of the hip using the Pavlik harness: long-term results. J Bone Joint Surg [Br] 2007;89-B:230-5.

9. Mostert AK, Tulp NJ, Castelein RM. Results of Pavlik harness treatment for neonatal hip dislocation as related to Graf's sonographic classification. J Pediatr Orthop 2000;20:306-10.

10. Lerman JA, Emans JB, Millis MB, et al. Early failure of Pavlik harness treatment for developmental hip dysplasia: clinical and ultrasound predictors. J Pediatr Orthop 2001;21:348-53.

11. Wilkinson AG, Sherlock DA, Murray GD. The efficacy of the Pavlik harness, the Craig splint and the von Rosen splint in the management of neonatal dysplasia of the hip: a comparative study. J Bone Joint Surg [Br] 2002;84-B:716-19.

12. Peled E, Bialik V, Katzman A, Eidelman N, Norman D. Treatment of Graf's ultrasound class III and IV hips using Pavlik's method. Clin Orthop 2008;466:825-9.

13. Tönnis D. Congenital dysplasia and dislocation of the hip in children and adults. Berlin: Springer-Verlag, 1987. Chapter 18.

14. Herring JA. Conservative treatment of congenital dislocation of the hip in the newborn and infant. Clin Orthop 1992;281:41-7.

15. Graf R. Classification of hip joint dysplasia by means of sonography. Arch Orthop Trauma Surg 1984;102:248-55.

16. Tönnis $\mathbf{D}$. Normal values of the hip joint for the evaluation of $x$-rays in children and adults. Clin Orthop 1976;119:39-47.

17. Suzuki S, Kashiwagi N, Kasahara Y, Seto Y, Futami T. Avascular necrosis and the Pavlik harness the incidence of avascular necrosis in three types of congenital dislocation of the hip as classified by ultrasound. J Bone Joint Surg [Br] 1996;78-B:631-5. 